side, the yellow result light will flash on, indicating the truth value of $K N p N q$ for $p=$ wrong and $q=$ wrong according to the left position of the switches for the variables $p$ and $q$ on the right side of the keyboard.

Johannes Gutenberg-Universität in Mainz,

Institut für Angewandte Mathematik,

Mainz, Saarstrasse 21, Germany

1. J. Lukasiewicz \& A. TArski, "Untersuchungen über den Aussagenkalkül," Comptes Rendus des Séances de la Société des Sciences et des Lettres de Varsovie, Cl. III, 23, p. 31-32, 1930; and PaUd Rosenbloom, The Elements of Mathematical Logic, New York, 1950.

2. Private Communication, not published. Angstl reported on his results in a seminar on logistics held in 1950 at the University of Munich by Professor W. Britzelmayr.

3. W. BURKHART, "Electrical analysis of truth functions"" Thesis.

4. ARthUR W. BURKS, DON W ARREN \& JeSSe B. Wright, "An analysis of a logical machine using parenthesis-free notation," MTAC, v. 8, 1954, p. 53-57.

\title{
Evaluation at Half Periods of Weierstrass' Elliptic Function with Rectangular Primitive Period-Parallelogram
}

\section{By Chih-Bing Ling}

The purpose of this paper is to evaluate the following Weierstrass' elliptic function at half periods [1],

$$
e_{1}=\varnothing\left(\omega_{1}\right), \quad e_{2}=\varnothing\left(\omega_{2}\right), \quad e_{3}=\varnothing\left(\omega_{3}\right),
$$

where $2 \omega_{1}$ and $2 \omega_{2}$ are double periods of the function and $\omega_{3}$ is defined by

$$
\omega_{1}+\omega_{2}+\omega_{3}=0 \text {. }
$$

This paper tabulates only the values of the function whose primitive periodparallelogram is a rectangle with $2 \omega_{1}=1$ and $2 \omega_{2}=a i$, where $a \geqq 1$.

The three functions in (1) form a set of distinct roots of the cubic [1]

$$
x^{3}-p x-q=0,
$$

where

$$
p=15 \sigma_{4}, \quad g=35 \sigma_{6},
$$

and

$$
\begin{aligned}
\sigma_{2 k} & =\sum_{m, n=-\infty}^{\infty} \frac{1}{\left(2 m \omega_{1}+2 n \omega_{2}\right)^{2 k}} \\
& =2 \sum_{m=1}^{\infty} \frac{1}{m^{2 k}}+2 \sum_{n=1}^{\infty} \sum_{m=-\infty}^{\infty} \frac{1}{(m+n a i)^{2 k}} .
\end{aligned}
$$

The accent on the summation sign denotes the omission of simultaneous zero values of $m$ and $n$ from the double summation.

The cubic (3) indicates that

Received April 7, 1958; in revised form, August 7, 1959. 


$$
e_{1}+e_{2}+e_{3}=0 \text {. }
$$

Also, since $e_{1}, e_{2}$ and $e_{3}$ are distinct, the discriminant $\left(4 p^{3}-27 q^{2}\right)$ of the cubic does not vanish. As will be seen later, in the present case both $\sigma_{4}$ and $\sigma_{6}$ are real and $\left(4 p^{3}-27 q^{2}\right)$ is positive. This implies that all the roots of the cubic are real.

The evaluation of $\sigma_{4}$ and $\sigma_{6}$ is facilitated by using the known relation [2]

$$
\cot x=\frac{1}{x}+\sum_{m=-\infty}^{\infty}\left(\frac{1}{m \pi+x}-\frac{1}{m \pi}\right)
$$

where the accent on the summation sign denotes the omission of the zero value of $m$ from the summation. By repeated differentiation of Equation (7) and substitution of $i x$ for $x$, it is found that

$$
\begin{aligned}
& \sum_{m=-\infty}^{\infty} \frac{1}{(m \pi+i x)^{4}}=\frac{2}{3 \sinh ^{2} x}+\frac{1}{\sinh ^{4} x} \\
& \sum_{m=-\infty}^{\infty} \frac{1}{(m \pi+i x)^{6}}=-\frac{2}{15 \sinh ^{2} x}-\frac{1}{\sinh ^{4} x}-\frac{1}{\sinh ^{6} x} .
\end{aligned}
$$

Hence we have

where

$$
\begin{aligned}
& \sigma_{4}=\frac{\pi^{4}}{45}+\frac{4 \pi^{4} K_{1}}{3 \sinh ^{2} \pi a} \\
& \sigma_{6}=\frac{2 \pi^{6}}{945}-\frac{4 \pi^{6} K_{2}}{15 \sinh ^{2} \pi a}
\end{aligned}
$$

$$
\begin{aligned}
& K_{1}=\sinh ^{2} \pi a \sum_{n=1}^{\infty}\left(\frac{1}{\sinh ^{2} n \pi a}+\frac{3}{2 \sinh ^{4} n \pi a}\right) \\
& K_{2}=\sinh ^{2} \pi a \sum_{n=1}^{\infty}\left(\frac{1}{\sinh ^{2} n \pi a}+\frac{15}{2 \sinh ^{4} n \pi a}+\frac{15}{2 \sinh ^{6} n \pi a}\right) .
\end{aligned}
$$

Consequently, we find

$$
\frac{4 p^{3}-27 q^{2}}{16 \pi^{12}}=\frac{5 K_{1}+7 K_{2}}{3 \sinh ^{2} \pi a}+\frac{100 K_{1}^{2}-147 K_{2}^{2}}{\sinh ^{4} \pi a}+\frac{2000 K_{1}^{3}}{\sinh ^{6} \pi a} .
$$

With the aid of known tables [3, 4], values of $K_{1}, K_{2}$, and then $\sigma_{4}, \sigma_{6}$ and $\left(4 p^{3}-27 q^{2}\right)^{1}$ are computed to $16 \mathrm{D}$ for $a=1(0.25) 2(1) 6$ and $\infty$ as shown in Table 1.

The subsequent evaluation of $e_{1}, e_{2}$, and $e_{3}$ requires the solution of the cubic (3). It appears that one of the roots, $e_{1}$, can be easily evaluated to $16 \mathrm{D}$ as shown in Table 2 by using Newton's method or otherwise, but difficulty arises in evaluating the other two roots for in most cases they are almost equal. However, they can be separated by forming a new cubic

$$
x^{3}+p^{\prime} x-q^{\prime}=0
$$

whose roots are the differences of the roots of the cubic (3). Let $\left(e_{1}-e_{2}\right),\left(e_{2}-e_{3}\right)$ and $\left(e_{3}-e_{1}\right)$ be the roots of the new cubic. We have

$$
\begin{aligned}
& p^{\prime}=\left(e_{1}-e_{2}\right)\left(e_{2}-e_{3}\right)+\left(e_{2}-e_{3}\right)\left(e_{3}-e_{1}\right)+\left(e_{3}-e_{1}\right)\left(e_{1}-e_{2}\right)=-3 p, \\
& {q^{\prime}}^{\prime 2}=\left(e_{1}-e_{2}\right)^{2}\left(e_{2}-e_{3}\right)^{2}\left(e_{3}-e_{1}\right)^{2}=4 p^{3}-2 T q^{2} .
\end{aligned}
$$



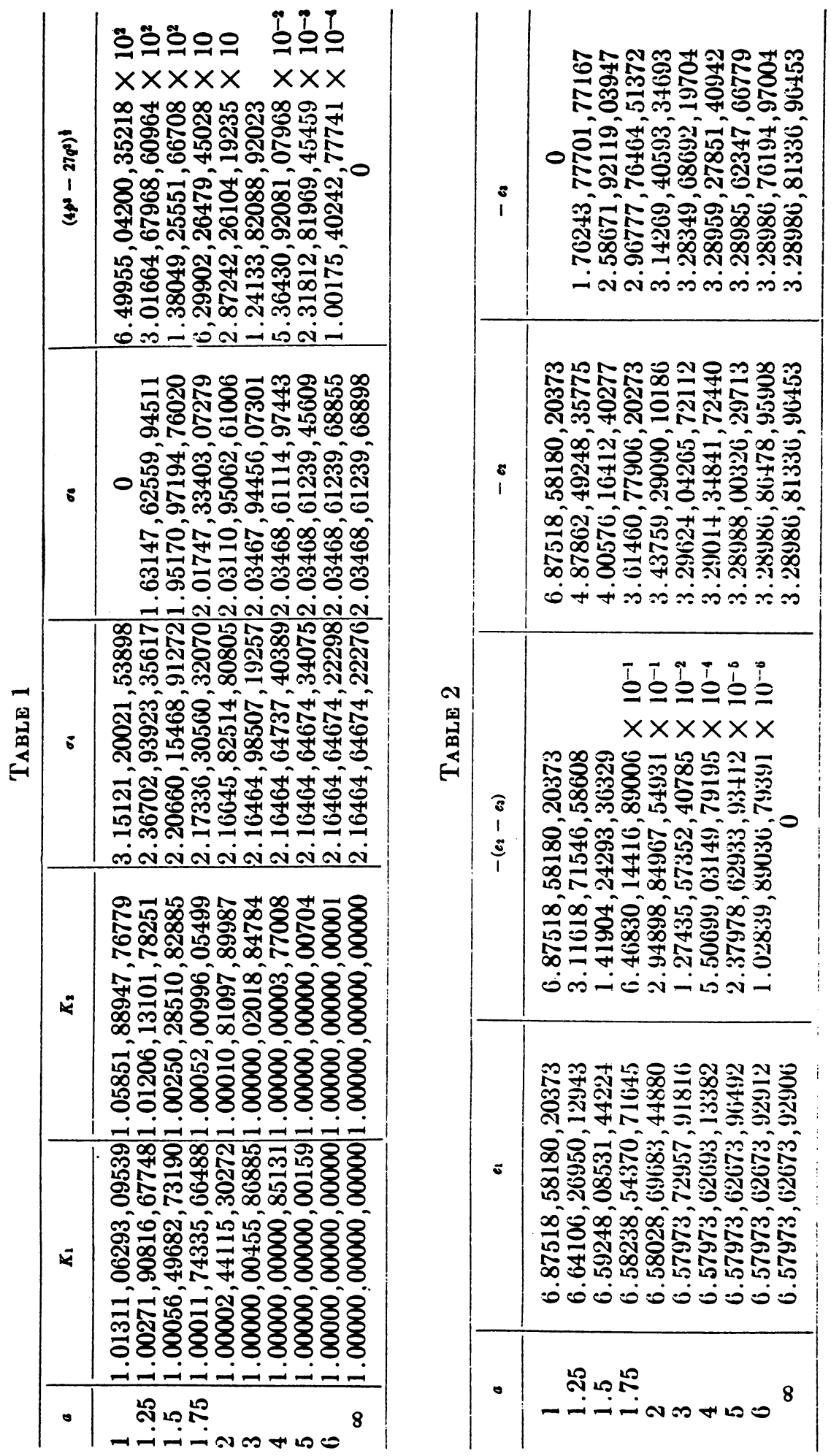
Consequently, by taking a positive sign for $q^{\prime}$, the new cubic is in the form

$$
x^{3}-3 p x-\left(4 p^{3}-27 q^{2}\right)^{3}=0 .
$$

From this cubic, values of $\left(e_{2}-e_{3}\right)$ and then $e_{2}$ and $e_{3}$ are computed to $16 D$ as shown in Table 2.

It is mentioned that the values of the function, for $0 \leqq a<1$ or in general for $\omega_{2} / \omega_{1}$ purely imaginary, can be computed from the tabulated values with the aid of the following relation [1]

$$
\wp\left(\lambda z \mid \lambda \omega_{1}, \lambda \omega_{2}\right)=\lambda^{-2} \wp\left(z \mid \omega_{1}, \omega_{2}\right)
$$

where $\lambda$ is a constant, real or complex.

The writer wishes to express his thanks to Mr. C. P. Tsai for his assistance in performing the numerical computations. The writer also is deeply grateful to Professor C. W. Nelson of the University of California, Berkeley, for checking the manuscript and verifying all the numerical values in Tables 1 and 2 by independent calculations. Thanks are also due to the referee of the paper, who suggests a different method of computation [5] without solving the cubic equation.

Institute of Mathematics

Academia Sinica

Taiwan, China

1. E. T. Copson, Theory of Functions of a Complex Variable, Oxford University Press, New York, 1935, p. 359-362.

2. E. P. ADAMS \& R. L. Hipristexy, Smithsonian Mathematical Formulae and Tables of Elliptic Functions, Smithsonian Miscellaneous Collection 74, Smithsonian Institution, Washington, D. C., 1939 , p. 129.

3. J. W. L. GLaisuER, "Tiables of $1 \pm 2^{-n}+3^{-n} \pm 4^{-n}+$ etc., and $1+3^{-n}+5^{-n}+7^{-n}+$ etc., to 32 places of decimals," Quart. Jn. Pure and Appl. Math., v. 45, 1914, p. 141-158.

4. British Association for the AdvanCEMent of Science, Mathematical Tables Volume 1, Circular and Hyperbolic Functions, elc., University Press, Cambridge, 1946, p. 24-29.

5. A. ERDELYI, ET AL., Higher Transcendental Functions, v. 2, McGraw-Hill Book Co., New York, 1953, p. 361 .

\section{A Note on the Nonexistence of Certain Pro- jective Planes of Order Nine}

\section{By Raymond B. Killgrove}

1. Introduction. Every finite projective plane may be coordinatized in at least one way [1]. In this process some lime is chosen to be the line at infinity, and the points not on this line are represented by an ordered pair of elements. The elements $x$ and $y$ for any point $(x, y)$ on a given line of the plane satisfy the equation $y=x \cdot m \circ b$, where $m$ and $b$ are specific elements for the given line. This ternary operation on $x, m$, and $b$ includes an additive loop in a special case.

A sequence of SWAC computer routines has been written to search for all planes having a specific additive loop in an appropriate ternary ring. Using these routines, a complete search had been made previously using the elementary Abelian group for the additive loop [2]. Now a complete search has been made using the

Received August 19, 1959. The work of Mr. Killgrove and the preparation of this paper were supported in part by the Office of Naval Research. 